\title{
Immunohistochemical characterisation of the costochondral junction in SIDS
}

\author{
R W Byard, B K Foster, S Byers
}

\begin{abstract}
Aim: To characterise the distribution of major growth plate proteoglycans in a group of infants who died of SIDS.

Methods: Nine such infants and eight age matched controls were selected from the necropsy files at the Adelaide Children's Hospital. Sections of rib and adjacent costal cartilage were stained for chondroitin-4-sulphate, chondroitin-6-sulphate, and keratan sulphate with antibodies 2-B-6, 3-B-3, and 5-D-4, respectively, using standard techniques.

Results: The distribution of proteoglycans within the extracellular matrix of the costochondral junction in the group of SIDS infants was identical with that found in control infants.

Conclusion: Any changes present in the growth plate in cases of SIDS are most likely caused by secondary phenomena.
\end{abstract}

$(\mathcal{F}$ Clin Pathol 1993;46:108-112)

It has been proposed that infants who die of sudden infant death syndrome (SIDS) have evidence of growth retardation ${ }^{12}$ including abnormalities of growth plate morphology. ${ }^{3}$ As there is a close and intricate association between growth plate chondrocytes and their surrounding matrix which they synthesise and modify in preparation for new bone deposition, ${ }^{4}$ an abnormality in the growth plate could be a primary phenomenon reflected in the composition of the cartilage extracellular matrix. The proteoglycan composition of the growth plate in SIDS has not, however, been extensively investigated.

In the following study a comparison of three carbohydrate epitopes found on the large cartilage proteoglycan, aggrecan, was undertaken to characterise more fully growth plate proteoglycan within the costochondral junction of a series of SIDS cases compared with controls.

\section{Methods}

Costochondral junctions of the sixth to eight ribs were taken at necropsy from nine infants in whom SIDS was subsequently diagnosed (age range 7 weeks-11 months; average $6 \cdot 3$ months), and from eight controls (age range 11 weeks-11 months; average $6 \cdot 1$ months who had died suddently from asphyxia (four cases: one inhalation of foreign body; two strangulation on clothing in cot; one strangulation on seat belt in car seat), trauma (two cases: one multiple trauma with head injury from a motor vehicle accident; one case of non-accidental injury), poisoning (one case of iatrogenic overdose of ethylnoradrenaline), and drowning (one case in the bath). The male:female ratio in the SIDS group was 4:5 and 5:3 in the control group. Five of the control infants were dead on arrival to hospital; two died within hours of resuscitation and one died within two days of resuscitation.

After routine processing, which included fixation in $10 \%$ formalin and decalcification in nitrous acid/EDTA, $5 \mu \mathrm{m}$ sections from the growth plates were cut and stained with haematoxylin and eosin, and with monoclonal antibodies to carbohydrate epitopes on the proteoglycan macromolecule, using standard methods. $^{56}$

Primary antibody reactivity was visualised using horseradish peroxidase conjugated sheep antimouse immunoglobulin (Silenus, Melbourne, Australia) and diaminobenzidine substrate (Sigma, St Louis, Missouri, USA). Sections minus first antibody incubation were included as negative controls, and Mayer's haematoxylin was used as a counterstain.

The antibodies 2-B-6, 3-B-3, and 5-D-4 (kindly provided by Professor B Caterson, University of North Carolina, Chapel Hill, USA) were used to identify specific glycosaminoglycan sequences in chondroitin-4-sulphate, chondroitin-6-sulphate, and keratan sulphate, respectively. Both $2-\mathrm{B}-6$ and $3-\mathrm{B}-3$ required chondroitinase $\mathrm{ABC}$ lyase (ICN Immunobiologicals, Costa Mesa, California, USA) digestion to expose epitopes. Sections were therefore digested with $0.05 \mathrm{IU} / \mathrm{ml}$ enzyme in $0 \cdot 1 \mathrm{M}$ TRIS-acetate buffer ( $\mathrm{pH} 7 \cdot 5$ ) for one hour at $37^{\circ} \mathrm{C}$ before the first antibody was added.

Growth plate morphology, on standard haematoxylin and eosin stained sections, and growth plate proteoglycan distribution, on immunohistochemical staining, were assessed for each case with no knowledge of the cause of death.

\section{Results}

Growth plates taken from cases of SIDS showed an orderly structure with regular col- 


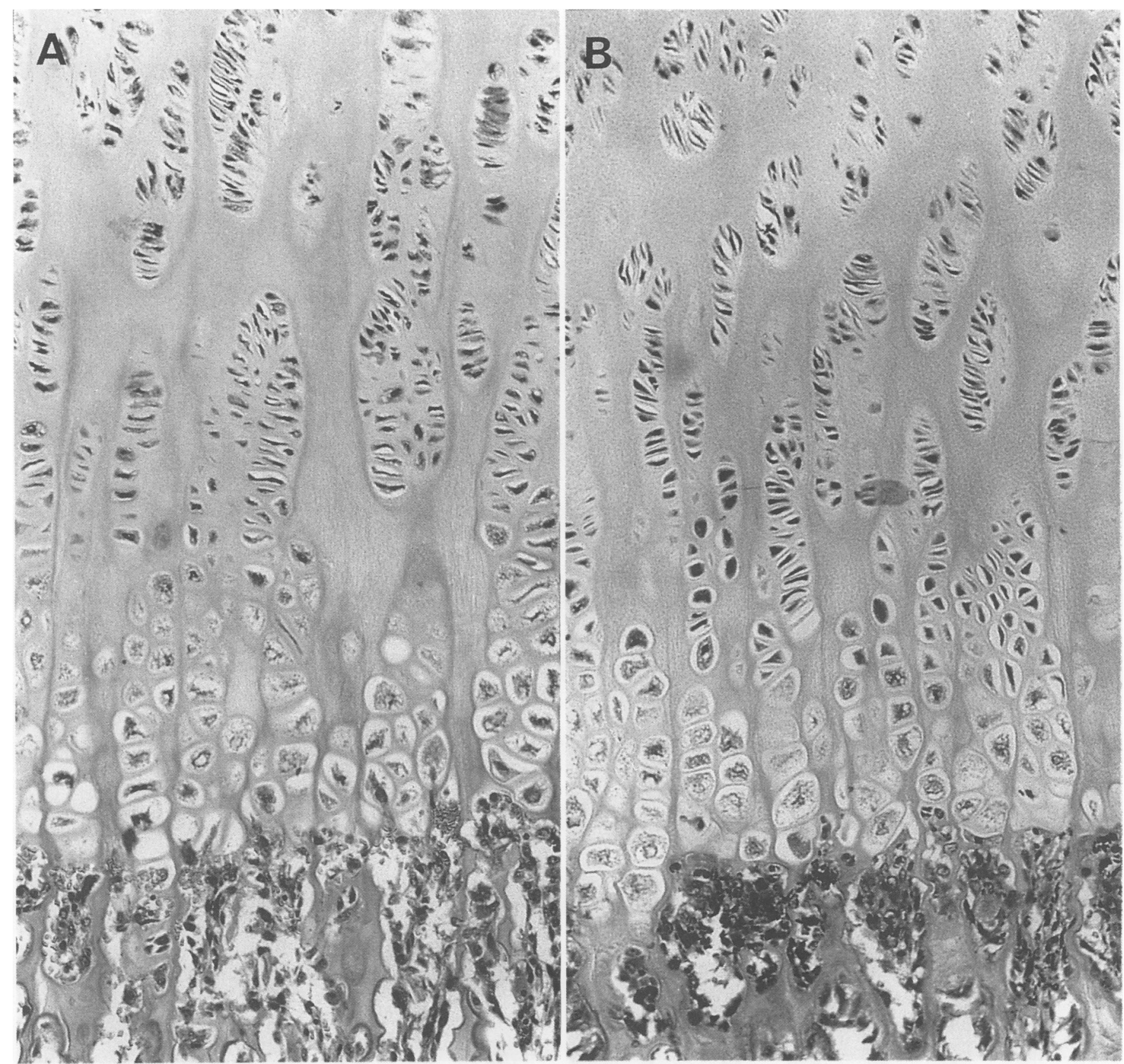

Figures $1 A$ and $B$ Orderly growth plate morphology showing columns of hypertrophic chondrocytes abutting marrow spaces where new bone is being laid down in $(A)$ SIDS infant and $(B)$ control infant (haematoxylin and eosin).

umns of chondrocytes, separated by extracellular matrix, undergoing progressive hypertrophy as they approached the metaphysis (fig 1A). No significant growth plate arrest, manifest by accumulation of matrix material between the most distal chondrocytes in the hypertrophic zone and the subjacent growing bone, or bridging of trabeculae between columns of chondrocytes, ${ }^{3}$ was identified. All of the control infants showed a similar orderly costochondral junction on assessment of the haematoxylin and eosin stained sections (fig 1B), except for one infant who had died of non-accidental injury and who had disorganisation and growth plate arrest suggestive of rickets.

Antibody staining for 2-B-6 demonstrated a widespread, even distribution of chondroitin- 4-sulphate throughout the growth plate of both SIDS and control cases (figs 2A and B). Staining was evident in the extracellular matrix, was closely associated with chondrocytes, and persisted in the calcified cartilage trabeculae of the metaphyseal bone.

Antibody staining for 3-B-3 showed an identical diffuse pattern (figs $3 \mathrm{~A}$ and $\mathrm{B}$ ) in the distribution of chondroitin-6-sulphate to 2-B-6 (chondroitin-4-sulphate).

Antibody staining for 5-D-4 demonstrated a zonal pattern with the presence of keratan sulphate glycosaminoglycan chains intimately associated with chondrocytes in the hypertrophic zone of the growth plate of both SIDS cases and controls (figs $4 \mathrm{~A}$ and $\mathrm{B}$ ). No staining was found in the calcified cartilage trabeculae that persist into the metaphyseal bone, 

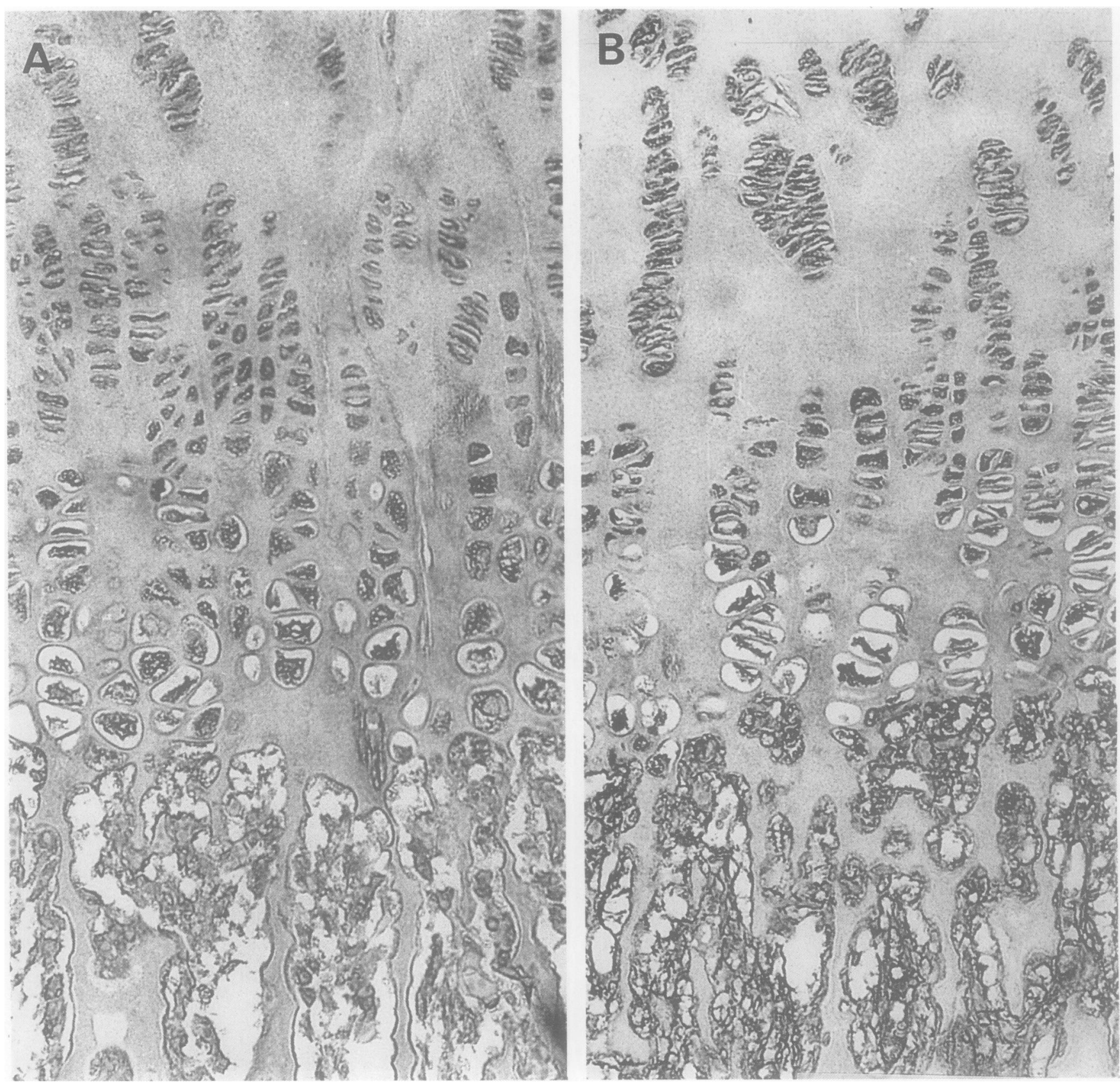

Figures $2 A$ and $B$ Uniform distribution of chondroitin-4-sulphate throughout the growth plate and into the metaphysis in both SIDS infant (A) and control (B) (haematoxylin and eosin).

emphasising the chondrocyte associated nature of this epitope.

The patterns of proteoglycan distribution in the growth plates taken from SIDS cases were virtually identical with those found in growth plates taken from controls.

\section{Discussion}

It has been suggested that at least $60 \%$ of infants who die of SIDS have evidence of growth retardation on examination of the costochondral junction. ${ }^{3}$ This is in keeping with the findings of other studies which have reported that SIDS infants show both intrauterine growth retardation ${ }^{17}$ and delayed postnatal development. ${ }^{28}$ Before a specific feature can be accepted as a secondary phenomenon, in this case secondary to overall delayed growth, it is appropriate to exclude primary abnormalities in the composition and expression of component tissues. This is exemplified by the debate as to whether surfactant abnormalities reported in SIDS are due to a primary difference in the nature of surfactant in these infants, or to dysfunction secondary to overheating or bacterial enzyme activity.

An intimate relation exists between extracellular matrix, changing chondrocyte morphology, and the process of ossification in the growth plate of the costochondral junction. Chondrocytes within each of the zones of the growth plate are responsible for the synthesis and organisation of surrounding matrix, which is composed primarily of large aggregating proteoglycans trapped in a network of type II collagen. ${ }^{4}$ As chondrocytes mature and hypertrophy, modulations in the composition of this extracellular matrix are observed. Regional variation in glycosaminoglycan content is 

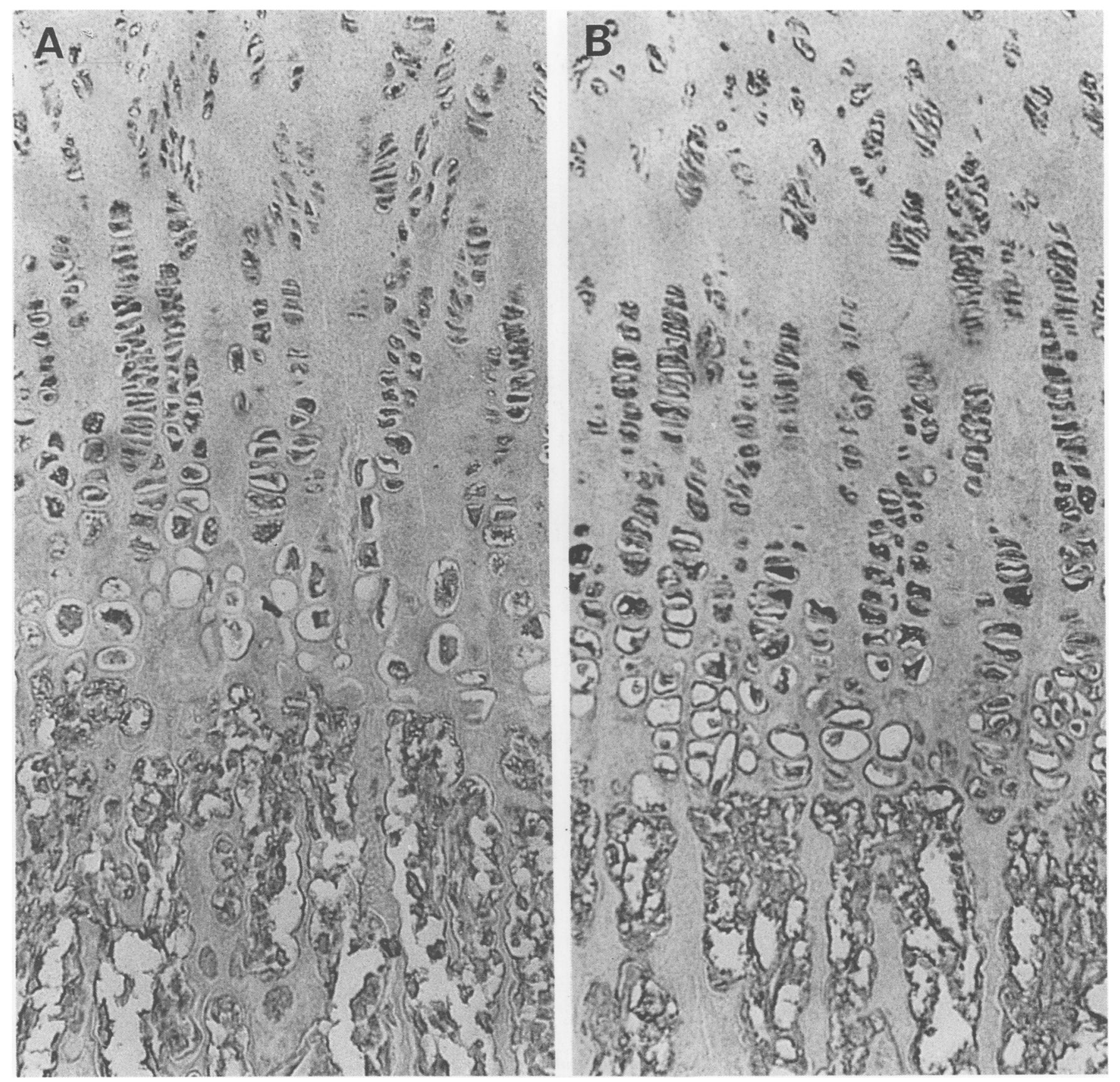

Figures $3 A$ and $B$ Uniform distribution of chondroitin-6-sulphate throughout the growth plate and into the metaphysis in both SIDS infant ( $A$ ) and control (B) (haematoxylin and eosin).

observed between the different zones, and within different areas of the same zones. Collagen content also changes with the appearance of a unique collagen type $X$ in the hypertrophic region. ${ }^{10}$ This continual and gradual modification of growth plate matrix reflects the variable functions of different growth plate regions, and it is only with the correct sequence of cell and matrix changes that the appropriate environment, which results in orderly mineralisation of the matrix in the hypertrophic zone prior to blood vessel invasion and new bone deposition, ${ }^{4}$ will be created.

It is therefore appropriate to characterise more clearly antigen expression in the macromolecular component of the extracellular matrix in order to help clarify the pathogenetic mechanisms that may be responsible for any altered morphology that is observed.
Immunohistochemical staining of three carbohydrate epitopes in this study showed no significant difference between SIDS cases and controls in the intensity and distribution of staining. This preliminary finding suggests that the abnormal growth plate morphology reported in a percentage of infants dying of SIDS is a secondary phenomenon, not related to primary skeletal dysfunction, and that there is no intrinsic abnormality of component proteoglycans within the costochondral junction.

This study was supported by the National Health and Medical Research Council of Australia, grant No $\mathbf{8 8 0 5 5 0 .}$

1 Buck GM, Cookfair DL, Michalek AM, Nasca PC, Standfast SJ, Sever LE, Kramer AA. Intrauterine growth retardation and risk of sudden

2 Williams SM, Taylor BJ, Ford RPK, Nelson EAS. Growth velocity before sudden infant death. Arch Dis Child velocity before sud
$1990 ; 65: 1315-8$.

3 Sinclair-Smith C, Dinsdale F, Emery J. Evidence of 

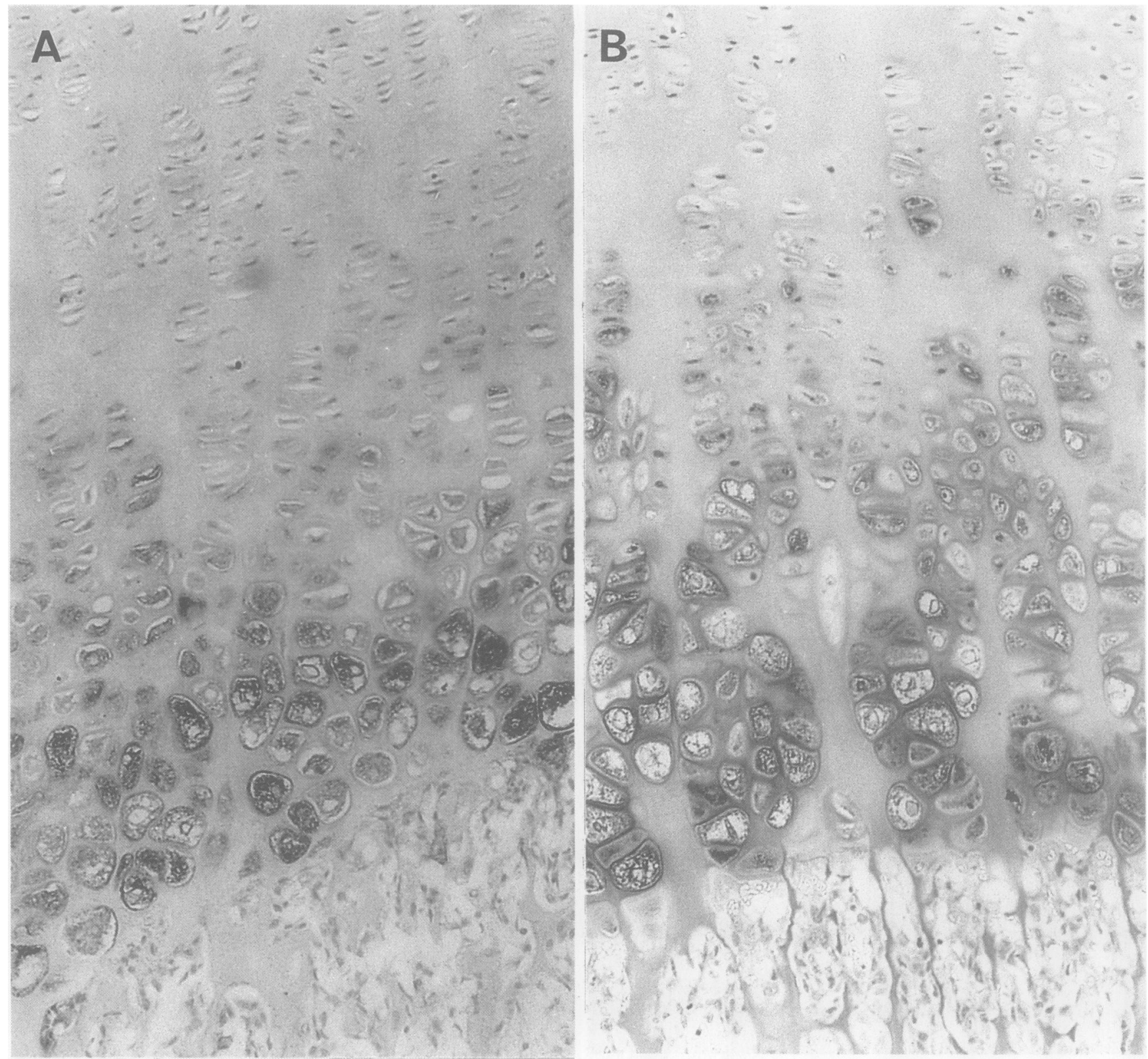

Figures $4 A$ and $B \quad$ Band-like distribution of keratan sulphate associated with cells within the hypertrophic zone, and not with extracellular matrix of the proliferative zone, or with cartilage matrix within the metaphysis in $(A)$ SIDS infant and (B) control.

duration and type of illness in children found unexpectedly dead. Arch Dis Child 1976;51:424-8.

4 Ogden JA, Rosenberg LC. Defining the growth plate. In: Uhthoff HK, Wiley JJ, eds. Behaviour of the growth plate. New York: Raven Press, 1988:1-15.

5 Byers S, Caterson B, Hopwood JJ, Foster BK. Immunolocation analysis of glycosaminoglycans in the human growth Cytochem 1992;40:275-82.

6 Sorrell JM, Lintala AM, Mahmoodian F, Caterson B. Epitope specific changes in chondroitin/dermatan sulfate proteoglycans as markers in the lymphopoietic and granulopoietic component of developing bursa of Fabriculus. F Immunol 1988;140:4263.

7 van Belle G, Hoffman HJ, Peterson DR. Intrauterine growth retardation and the sudden infant death syndrome. In: Harper RM, Hoffman HJ, eds. Sudden infant death syndrome. Risk factors and basic mechanisms. New death syndrome. Risk factors and basic

8 Peterk: PR Publishing, Fisher JB. Postnatal growth and the sudden infant death syndrome. Am $\mathcal{Y}$ Epidemi 1974;99:389-94.

9 Byard RW. Possible mechanisms responsible for the sudden infant death syndrome. F Paediatr Child Health 1991, 27:147-57

10 Gibson GJ, Francki KT, Hopwood JJ, Foster BK. Human and sheep growth plate cartilage type $\mathrm{X}$ collagen synthesis and the influence of tissue storage. Biochem $\mathcal{F}$ 1991;277: 513-20. 